\title{
The nonlinear optical loop mirror: soliton and noise-like pulse emission in a figure-eight fiber laser
}

\author{
A. Camarillo-Avilés ${ }^{a}$, E. Hernández-Escobar ${ }^{a}$, R. López-Estopíer ${ }^{a, b}$, M. Bello-Jiménez ${ }^{a, *}$, \\ O. Pottiez ${ }^{c}$, B. Ibarra- Escamilla ${ }^{d}$, M. Duran-Sánchez ${ }^{b, d}$, and M. V. Andrés ${ }^{e}$ \\ ${ }^{a}$ Instituto de Investigación en Comunicación Óptca, Universidad Autónoma de San Luis Potosí, \\ Av. Karakorum N. 1470 Lomas 4 a Secc., 78210 San Luis Potosí, Mexico. \\ ${ }^{b}$ Consejo Nacional de Ciencia y Tecnología, Av. Insurgentes Sur No. 1582, Col. Crédito, \\ Constructor, Del. Benito Juárez, México, 039040, Ciudad de México. \\ ${ }^{c}$ Centro de Investigaciones en Óptica, \\ Loma del Bosque 115, Col. Lomas del Campestre, León, Gto. 37150, Mexico. \\ ${ }^{d}$ Instituto Nacional de Astrofísica, Óptica y Electrónica, \\ Luis Enrique Erro N. 1, Departamento de Óptica,72000 Puebla, Mexico. \\ ${ }^{e}$ Universidad de Valencia, Departamento de Física Aplicada y Electromagnetismo, \\ ICMUV, c/Dr. Moliner 50, Burjassot, 46100 Valencia, Spain. \\ *e-mail: miguel.bello@uaslp.mx
}

Received 28 November 2020; accepted 22 December 2020

\begin{abstract}
In this article, a symmetrical nonlinear optical loop mirror (NOLM) exhibiting a polarization-dependent transmission is evaluated to generate optical pulse emission in a figure-eight fiber laser in the soliton and noise-like pulse (NLP) regimes. The NOLM structure relies on a 50:50 fiber coupler, a loop with highly twisted single-mode optical fiber and a quarter-wave retarder (QWR) to break the polarization asymmetry. The pulse operation regime is determined by properly adjusting the NOLM low-power transmission, which is easily realized by the rotation of the QWR angle. Soliton pulses of 1.48 ps pulse duration and peak power of $18 \mathrm{~W}$ were observed with a peak to peak separation of 1.25 $\mu \mathrm{s}$, corresponding to a fundamental cavity repetition rate of $0.8 \mathrm{MHz}$. Moreover, by incrementing the NOLM low-power transmission, NLP emission is generated, exhibiting a wide and smooth spectrum of $8.5 \mathrm{~nm}$ bandwidth.
\end{abstract}

Keywords: Erbium doped fiber; soliton pulse; noise-like pulse; nonlinear optical loop mirror; fiber lasers.

PACS: 42.55.Wd; 42.60.-v; 42.65.-k

\section{Introduction}

Passively mode-locked fiber lasers producing ultrashort optical pulses have become essential optical light sources with several practical applications. Nowadays, new configurations of passively mode-locked fiber lasers are still suggested following the demands for high-quality ultrashort pulse generation [1-6]. Among these approaches, the so-called "figure of eight" fiber laser (F8L) has emerged as one of the most promising laser architectures to investigate pulse dynamics in fiber lasers. Besides the proper adjustment of intracavity parameters allows a laser emission under different pulse regimes. These include, for example, the soliton-like pulse [7,8], dissipative soliton resonance [9-13], bound soliton [1416], soliton cluster [17,18], and noise-like pulse (NLP) [1922].

In this work, a symmetrical nonlinear optical loop mirror (NOLM) with polarization-dependent transmission is analyzed in a F8L structure to generate soliton and NLP emission. The NOLM consists of a 50:50 fiber coupler, a loop composed of 220-m long twisted single-mode fiber and a quarter-wave retarder (QWR) located asymmetrically in the NOLM loop. As shown in the next sections, the NOLM performs a similar operation than a half-wave retarder in the low-power regime, allowing the transmission of a polariza- tion state that is orthogonal to the input. In this way, the parallel component undergoes a zero transmission in the lowpower regime and nonzero transmission only at high power levels. These characteristics of the NOLM will allow a precise control on the mode locking process by adjusting the transmission of light to the cavity via the low-power transmission, which is achieved in our setup by the rotation of the QWR angle. The experimental results demonstrate a soliton pulse emission of 1.48 ps pulse duration and $18 \mathrm{~W}$ of peak power at the fundamental cavity repetition rate of 0.8 MHz. Besides by a slight increment of the QWR angle, NLP emission is generated, exhibiting a broad and smooth optical spectrum of $8.5 \mathrm{~nm}$ in bandwidth.

\section{The NOLM configuration}

A schematic view of the NOLM is illustrated in Fig. 1. The NOLM comprises a symmetrical coupler, a loop of highly twisted SMF-28 fiber and a QWR located asymmetrically in the loop. A twist rate of 7 turns per meter was set to the fiber in the loop. This modification allows a significant reduction of the residual linear birefringence and preserves the polarization state $[23,24]$. The location of the QWR is immediately after the port P4, in such a way that the beam traveling in a counterclockwise sense, from port P4 to port P3, 


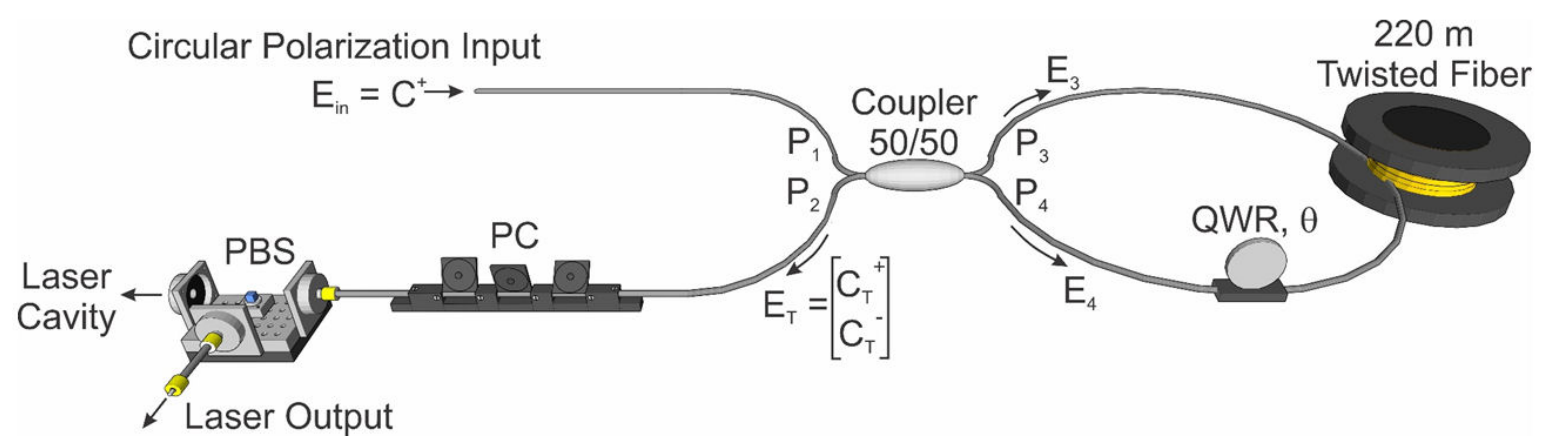

FIGURE 1. The NOLM setup.

changes its polarization state when starting its propagation along the loop, whereas the beam traveling in the opposite sense (from port P3 to port P4) undergoes a change of polarization state when completes its trajectory. For the particular case of circular right input polarization, the NOLM transmission in a low-power regime can be adjusted for values between 0 and 0.5 through a rotation of the QWR angle while maintaining a nonlinear transmission close to 1 at the critical power in any case [25]. For this reason, the NOLM is analyzed considering a circular right input polarization. A detailed description of the NOLM operation under different polarization input states can be found in $[25,26]$. For this arrangement, a polarization beam splitter (PBS) cube is introduced after the NOLM output for monitoring the transmitted light.

The NOLM is analyzed considering each of the polarization states that compose the total field at the NOLM output, see Fig. 1. $E_{\text {in }}$ represents the input field with a circular right polarization $C^{+}, E_{3}$ and $E_{4}$ are the fields transmitted by the coupler via the ports $P_{3}$ and $P_{4}$, respectively, the rotation angle of the QWR is represented by $\theta$, and total field $E_{T}$ denotes the transmitted field at the NOLM output. Note that $E_{T}$ is composed by two orthogonal polarization states, $C_{T}^{+}$ and $C_{T}^{-}$. The total transmitted field $E_{T}$ is expressed by the following equation,

$$
\begin{aligned}
\left|E_{T}\right|^{2} & =\left[\begin{array}{c}
\left|C_{T}^{+}\right|^{2} \\
\left|C_{T}^{-}\right|^{2}
\end{array}\right] \\
& =\left[\begin{array}{c}
\frac{\left|E_{i n}\right|^{2}}{2}\left(1-4 \alpha[1-\alpha] \cos ^{2}\left[\frac{\Delta \varphi_{N L}}{2}\right]\right) \\
\frac{\left|E_{i n}\right|^{2}}{2}\left(1-4 \alpha[1-\alpha] \cos ^{2}\left[\frac{\Delta \varphi_{N L-4 \theta}}{2}\right]\right)
\end{array}\right],
\end{aligned}
$$

where $\Delta \varphi_{N L}$ represents the nonlinear phase difference and $\alpha$ denotes the coupling ratio of the coupler ( $\alpha=0.5$ for a symmetrical coupler).

Assuming the NOLM is operated under a low-power regime, the $\Delta \varphi_{N L}$ is equal to zero, and the output field $C_{T}^{+}$, with a polarization state that is parallel to the input, undergoes a null transmission. On the contrary, the transmitted field $C_{T}^{-}$, whose polarization state is orthogonal to the input, experiences a nonzero transmission due to its dependence on the rotation angle $\theta$. This feature of the NOLM describes a

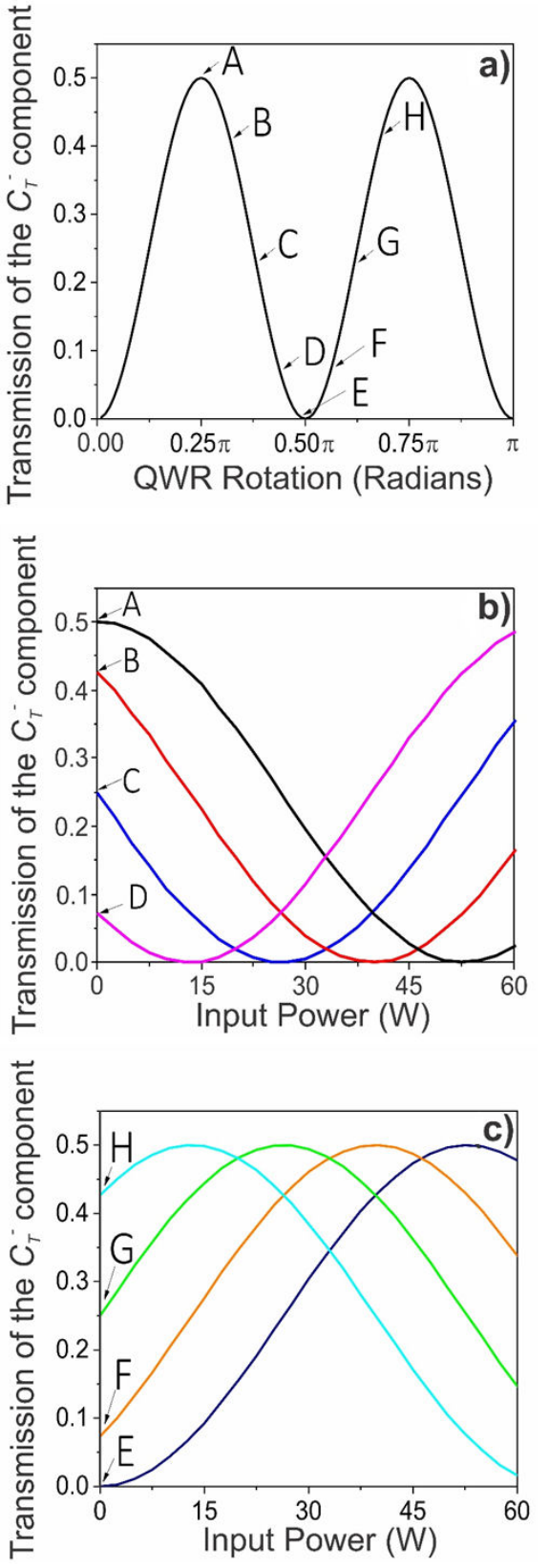

FIGURE 2. a) Low-power transmission of the NOLM as a function of the QWR rotation angle. Nonlinear transmission of the $C_{T}^{-}$ component at the angles b) $A-D$ and c) $E-H$ of the QWR. 


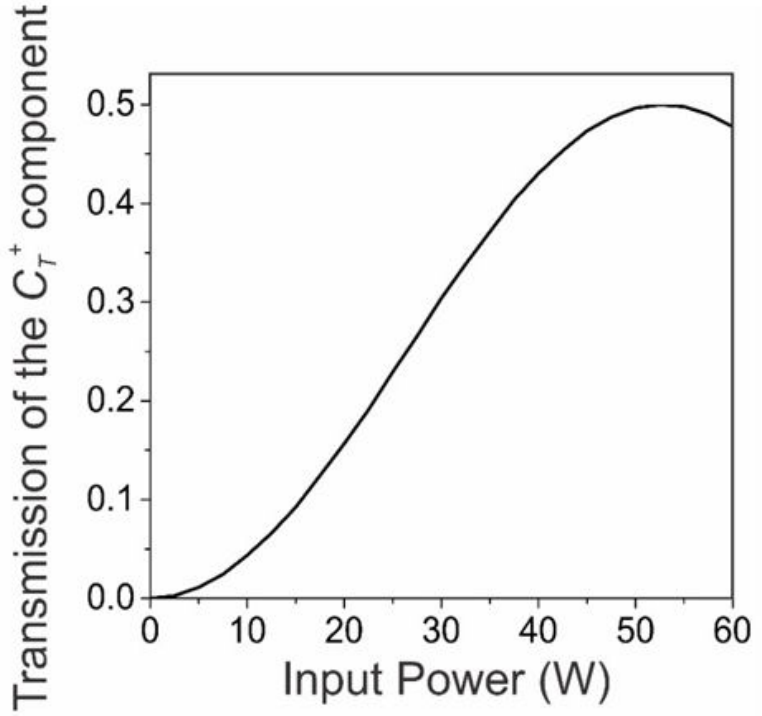

FIGURE 3. Nonlinear transmission of the $C_{T}^{+}$component.

fluctuating transmission for the $C_{T}^{-}$component that depends on the orientation of the QWR angle, as shown in Fig. 2a), where the NOLM can be assumed to operate similarly a halfwave retarder in the low-power regime. Figure $2 b$ ) and 2c) show the nonlinear transmission of the NOLM for the $C_{T}^{-}$ component, where different QWR rotation angles were considered. The rotation angles are related to the points $A$ to $H$, as depicted in Fig. 2a).

From this set of transmission curves, the importance of the QWR angle is emphasized to accurately control the nonlinear transmission of the NOLM.

In a high-power regime, the nonlinear phase difference $\Delta \varphi_{N L}$ is different from zero and the $C_{T}^{+}$component emerges with a transmission that is not dependent on the rotation angle $\theta$, see Fig. 3. Therefore, it maintains fixed for any rotation of the QWR and performs a nonlinear response that is started from a zero level. This characteristic makes this NOLM transmission curve a very attractive solution to gener- ate optical pulses without low-intensity radiation or pedestals in a fiber laser system.

To take advantage of the NOLM properties in a F8L scheme, a polarization controller (PC) followed by a PBS cube is introduced after the NOLM output, see Fig. 1. The PC can be adjusted to provide a maximum transmission through the PBS in such a way that the low-power component can be transmitted to the laser cavity to initiate the mode locking operation. With this adjustment, the parallel polarization component, associated with high intensity radiation, is obtained through the output port of the PBS cube.

\section{The figure-eight fiber laser}

The schematic setup of the F8L is illustrated in Fig. 4. It is based on a symmetrical NOLM with polarization-dependent transmission. The characteristics of the NOLM are the same to that mentioned in the previous section. To take advantage of the NOLM properties, a polarization controller (PC3) connected to a PBS cube is adjusted in the low-power regime to provide the maximum transmission of light to the laser cavity, this allows that a small amount of energy will be amplified and reinserted to the NOLM input to facilitate the selfstarting mode-locking process. The remaining transmission port of the PBS is used to obtain the output light pulses, with a polarization state that is associated with high-intensity radiation. Hence, this configuration ensures an optical pulse operation without the contribution of a continuous-wave (cw) background. A 3-m long erbium-doped fiber (EDF) is used as the gain medium. It is pumped via the wavelength division multiplexer coupler (WDM) by a $975-\mathrm{nm}$ pigtailed laser diode, which delivers a maximum pump power of $200 \mathrm{~mW}$. A fiber isolator (ISO) is inserted to force unidirectional operation in the cavity. Finally, the polarization controllers, PC1 and $\mathrm{PC} 2$, are included to accurately control the polarization state at the NOLM input. The PC1 is adjusted to provide a maximum transmission through the linear polarizer (LP), and the $\mathrm{PC} 2$ is used to introduce a right circular polarization state at the NOLM input.

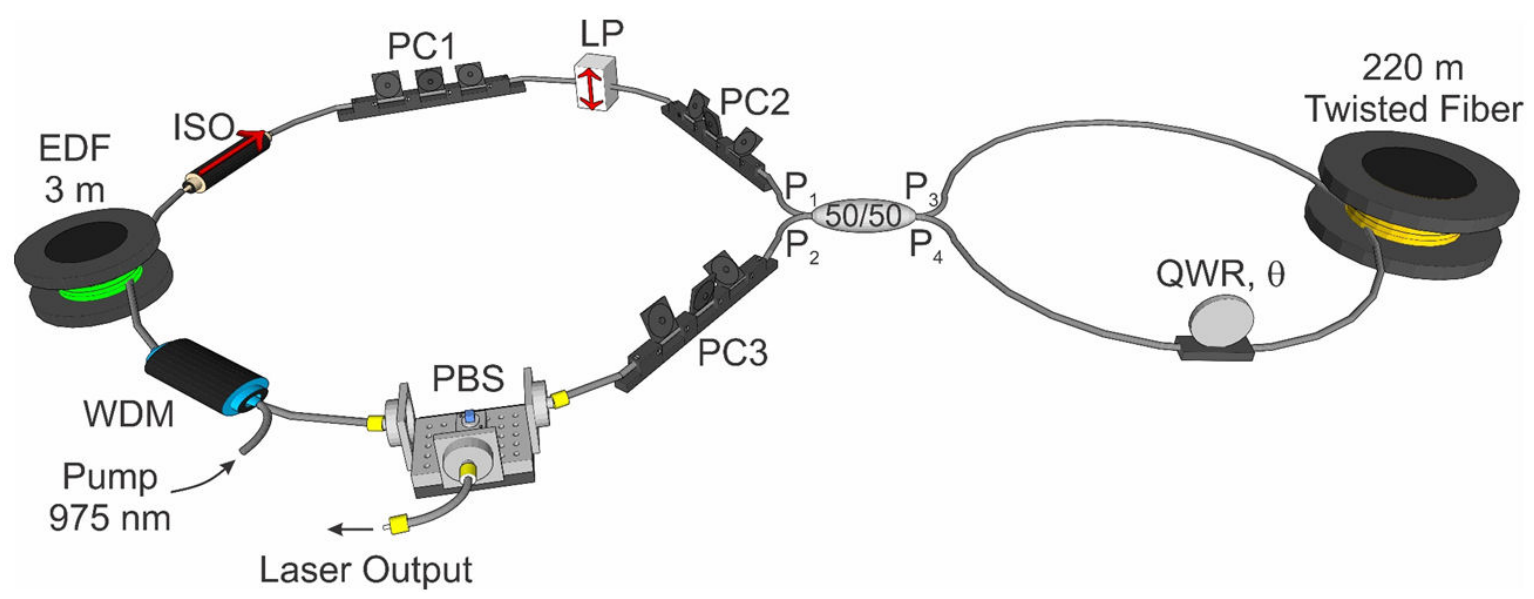

FIGURE 4. Schematic setup of the mode-locked figure-eight fiber laser. 

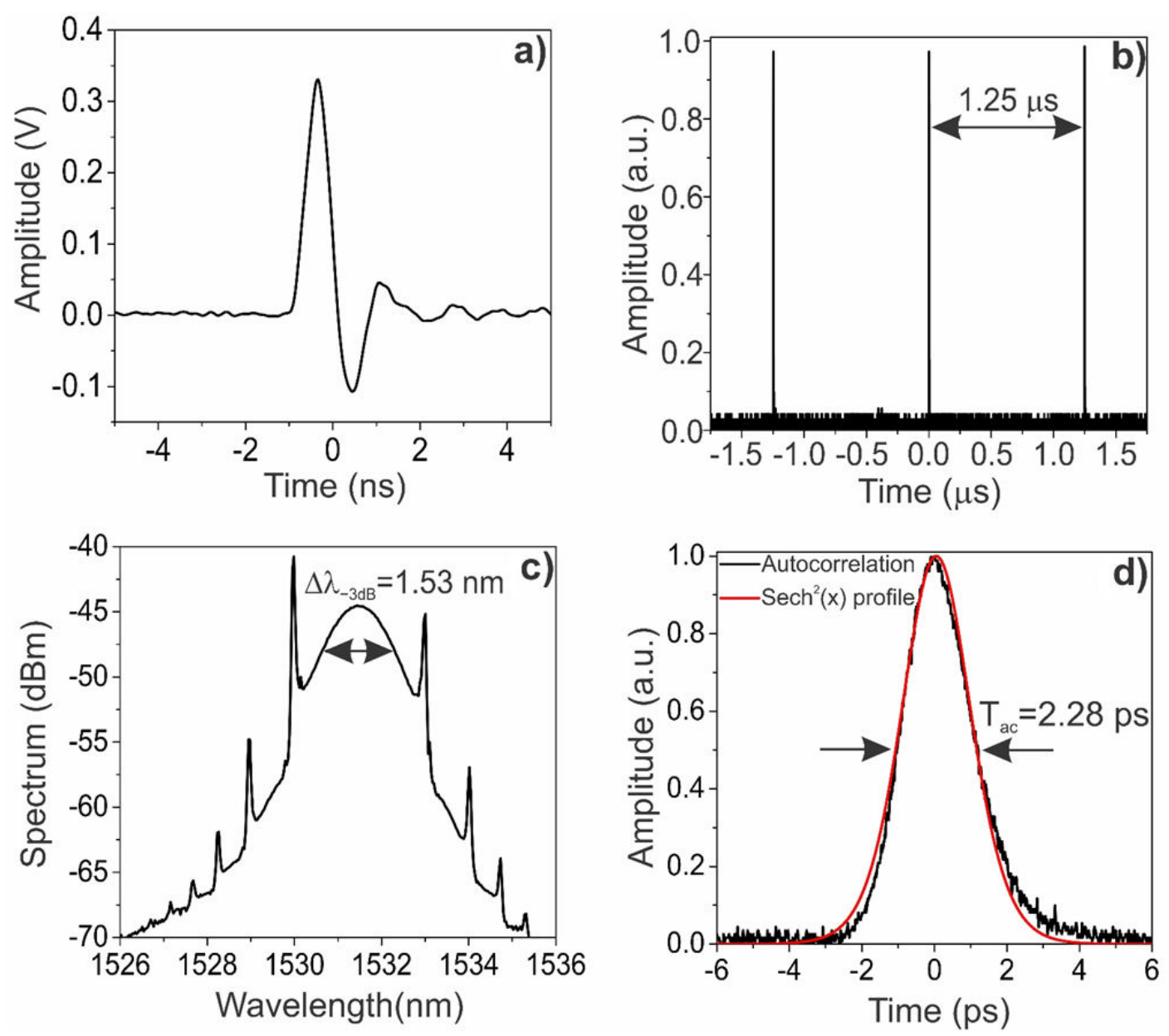

FIgURE 5. F8L operation in single-pulse regime. a) Pulse waveform monitored with a $1.2 \mathrm{GHz}$ photodetector. b) Mode-locked train of pulses with a peak to peak separation of $125 \mu \mathrm{s}$. c) Measured optical spectrum of the laser. d) Autocorrelation trace of a single mode-locked pulse.

Passively mode-locking operation was obtained through adjustments of the NOLM transmission via the QWR angle. The required angle to achieve a self-starting mode-locked pulse emission is determined at the region of positive slope in the low-power NOLM transmission curve. In our arrangement, it was found when the transmission was fixed to a value of 0.05 . At a pump power of around $40 \mathrm{~mW}$, a train of multiple mode-locked pulses appears, but if the pump power decreases, single-pulse emission is obtained at $9.5 \mathrm{~mW}$ of pump power. The average output power for single-pulse emission is $24 \mu \mathrm{W}$. Figure 5a) shows the output pulses measured with a $1.2 \mathrm{GHz}$ photodetector and a $2.5 \mathrm{GHz}$ oscilloscope. Although the short pulse cannot be precisely resolved in this measurement, we observe no contributions of $\mathrm{cw}$ radiation in the pulses. The pulse repetition frequency is shown in Fig. 5b); it was measured as $0.8 \mathrm{MHz}$. Thus, a cavity length of $259.28 \mathrm{~m}$ is estimated by considering an effective refractive index $\left(n_{e f f}\right)$ of 1.4463 . The measured optical spectrum of the laser is shown in Fig. 5c). The full width at half maximum (FWHM, $-3 \mathrm{~dB}$ on a logarithmic scale) spectrum bandwidth $(\Delta \lambda)$ is $1.53 \mathrm{~nm}$, centered at $1531.5 \mathrm{~nm}$ measured through the Yokogawa AQ6375 optical spectrum analyzer with 50-pm resolution. The spectrum power exhibits sharp Kelly sidebands. It confirms that the mode-locked pulses are solitons [27]. Figure 5d) shows the corresponding autocorrelation function, measured with a Femtochrome FR-103XL autocorrelator. A pulse duration $\left(T_{\mathrm{FWHM}}\right)$ of $1.48 \mathrm{ps}$ is calculated from the $2.28 \mathrm{ps}$ FWHM duration of the autocorrelation trace $\left(T_{a c}\right)$, i.e., $T_{\mathrm{FWHM}}=0.648 \mathrm{Tac}$. This measurement allows an estimation of the peak power as $18 \mathrm{~W}$. The timebandwidth product (TBP) was measured as 0.289 , close to the ideal transform-limit value of 0.315 for a squared hyperbolic secant pulse profile.

After the solitons observation our next step was to vary slightly the position of QWR into the NOLM loop. This adjustment was realized maintaining the polarization controllers and pump power fixed, and only at the proper orientation of the QWR angle, the mode-locked spectrum broadened and the Kelly sidebands disappear. This behavior is observed in our setup by incrementing the QWR angle to reach a low-power transmission of 0.1. Figure 6a) depicts the output spectrum, where it can be deduced from its spectral shape, a 

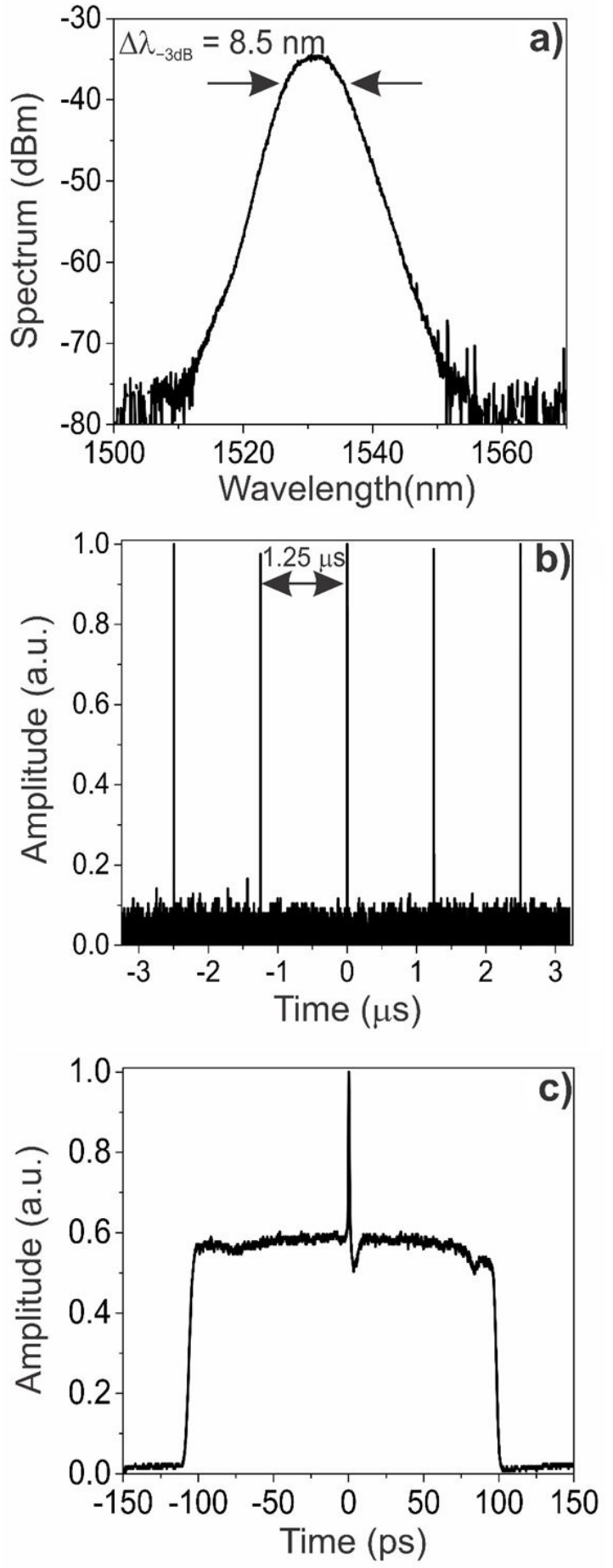

FIGURE 6. Noise-like pulse operation. a) Measured optical spectrum of the laser. b) Train of pulses generated at $0.8 \mathrm{MHz}$ with 73 $\mathrm{mW}$ of pump power. c) Autocorrelation trace. smooth and wide optical spectrum, that the F8L operates in the NLP regime. A pump power of $73 \mathrm{~mW}$ was necessary to achieve a stable train of NLPs. The spectrum reveals a -3 $\mathrm{dB}$ optical bandwidth of $8.5 \mathrm{~nm}$ with a central wavelength of $1531.5 \mathrm{~nm}$. The corresponding train of NLPs is depicted in Fig. 6b), where the fundamental cavity repetition rate of $0.8 \mathrm{MHz}$ is observed. An average output power of $0.96 \mathrm{~mW}$ is measured. Figure 6c) shows the autocorrelation trace for the NLPs, where a narrow coherent peak and wide shoulders are observed. These results are in good agreement with the intrinsic characteristics of the previously reported NLP fiber lasers [19-22].

The above results demonstrate that, by a simple and clear adjustment of the QWR it is possible to operate a modelocked F8L in the soliton and the NLP regimes. A solitonlike pulse emission is observed when the QWR angle is adjusted to produce a NOLM low-power transmission close to zero. This enables that a small amount of light at the NOLM output will be amplified and feedback to the NOLM input to initiate the mode-locking process. Besides if now the QWR is adjusted to obtain a higher NOLM low-power transmission, a single pulse is unstable and evolves toward a noise-like pulse operation. In both reported cases, we emphasize the absence of peaks or pedestals associated to a cw radiation.

\section{Conclusion}

A symmetrical NOLM with a polarization-dependent transmission was analyzed to generate optical pulse emission in a F8L. The results demonstrate that, by a simple and clear adjustment of the QWR, it is possible to obtain a modelocking operation in both the soliton and the noise-like pulse regimes. A single pulse operation is obtained when the QWR is adjusted to have a NOLM low-power transmission around 0.05. But, if the QWR is adjusted to obtain a higher lowpower NOLM transmission of 0.1 , the single pulse operation evolves toward a noise-like pulse.

\section{Acknowledgments}

In memory of Dr. Evgeny Kuzin, a great mentor and dear friend.

This work was supported by CONACyT "Ciencia de Frontera 2019" under Grant 217560 and FAI-UASLP project C20-FAI-10-28.28.
1. Xin Zou, Jifang Qiu, Xiaodong Wang, Zi Ye, Jindan Shi, and Jian $\mathrm{Wu}$, Versatile mode-locked fiber laser with switchable operation states of bound solitons, Appl. Opt. 55 (2016) 4323, https://doi.org/10.1364/AO.55.004323.
2. Ai-Ping Luo, Zhi-Chao Luo, Hao Liu, Xu-Wu Zheng, QiuYi Ning, Nian Zhao, Wei-Cheng Chen, and Wen-Cheng Xu, Noise-like pulse trapping in a figure-eight fiber laser, Opt. Express 23 (2015) 10421, https://doi.org/10.1364/ 
OE.23.010421.

3. J. Szczepanek, T. M. Kardás, M. Michalska, C. Radzewicz, and Y. Stepanenko, Simple all-PM-fiber laser mode-locked with a nonlinear loop mirror, Opt. Lett. 40 (2015) 3500, https : //doi.org/10.1364/OL.40.003500.

4. Jianfeng Li et al., Thulium-doped all-fiber mode-locked laser based on NPR and $45^{\circ}$-tilted fiber grating, Opt. Express 22 (2014) 31020, https : / / doi .org/10.1364/OE.22. 031020 .

5. Sheng-Fong Lin, Huai-Yung Wang, Yu-Chuan Su, Yu-Chieh Chi and Gong-Ru Lin, Multi-order bunched soliton pulse generation by nonlinear polarization rotation mode-locking erbium-doped fiber lasers with weak or strong polarizationdependent loss, Laser Phys. 24 (2014) 1, https://doi. org/10.1088/1054-660X/24/10/105113.

6. Zhi-Chao Luo, Qiu-Yi Ning, Hai-Lan Mo, Hu Cui, Jin Liu, LiJun $\mathrm{Wu}$, Ai-Ping Luo, and Wen-Cheng $\mathrm{Xu}$, Vector dissipative soliton resonance in a fiber laser, Opt. Express 21 (2013) 10199, https://doi.org/10.1364/OE.21.010199.

7. I. N. Duling III, All-fiber ring soliton laser mode locked with a nonlinear mirror, Opt. Lett. 16 (1991) 539, https : / / doi. org/10.1364/OL.16.000539.

8. V. J. Matsas, D. J. Richardson, T. P. Newson, and D. N. Payne, Characterization of a self-starting, passively mode-locked fiber ring laser that exploits nonlinear polarization evolution, Opt. Lett. 18 (1993) 358, https://doi.org/10.1364/OL. 18.000358 .

9. W. Chang, A. Ankiewicz, J. M. Soto-Crespo, and N. Akhmediev, Dissipative soliton resonances, Phys. Rev. A 78 (2008) 023830, https://doi.org/10.1103/PhysRevA. 78 . 023830 .

10. Ph. Grelu, W. Chang, A. Ankiewicz, J. M. Soto-Crespo, and N. Akhmediev, Dissipative soliton resonance as a guideline for high-energy pulse laser oscillators, J. Opt. Soc. Am. B 27 (2010) 2336, https://doi.org/10.1364/JOSAB. 27 . 002336 .

11. X. Wu, D. Y. Tang, H. Zhang, and L. M. Zhao, Dissipative soliton resonance in an all-normal-dispersion erbium-doped fiber laser, Opt. Express 17 (2009) 5580, https://doi.org/ $10.1364 / \mathrm{OE} .17 .005580$.

12. X. Li, X. Liu, X. Hu, L. Wang, H. Lu, Y. Wang, and W. Zhao, Long-cavity passively mode-locked fiber ring laser with high-energy rectangular-shape pulses in anomalous dispersion regime, Opt. Lett. 35 (2010) 3249, https : / / doi .org/10. $1364 /$ OL.35.003249.

13. Z. C. Luo, W. J. Cao, Z. B. Lin, Z. R. Cai, A. P. Luo, and W. C. $\mathrm{Xu}$, Pulse dynamics of dissipative soliton resonance with large duration-tuning range in a fiber ring laser, Opt. Lett. 37 (2012) 4777, https://doi.org/10.1364/OL.37.004777.

14. B. A. Malomed, Bound solitons in the nonlinear SchrodingerGinzburg-Landau equation, Phys. Rev. A, Mol. Opt. Phys. 44 (1991), 6954, https://doi.org/10.1007/ 3-540-54899-8_48.
15. D. Y. Tang, W. S. Man, H. Y. Tam, and P. D. Drummond, Observation of bound states of solitons in a passively modelocked fiber laser, Phys. Rev. A 64 (2001) 033814, https: //doi.org/10.1103/PhysRevA.64.033814.

16. P. Grelu, F. Belhache, F. Gutty, and J. M. Soto-Crespo, Phaselocked soliton pairs in a stretched-pulse fiber laser, Opt. Lett. 27 (2002) 966, https://doi.org/10.1364/OL.27. 000966 .

17. F. Amrani, M. Salhi, P. Grelu, H. Leblond, and F. Sanchez, Universal soliton pattern formations in passively mode-locked fiber lasers, Opt. Lett. 36 (2011) 1545, https://doi.org/10. 1364 /OL.36.001545.

18. F. Amrani, M. Salhi, H. Leblond, A. Haboucha, and F. Sanchez, Intricate solitons state in passively modelocked fiber lasers, Opt. Express 19 (2011) 13134, https://doi.org/10. 1364 /OE.19.013134.

19. M. Horowitz, Y. Barad, and Y. Silberberg, Noiselike pulses with a broadband spectrum generated from an erbium-doped fiber laser, Opt. Lett. 22 (1997) 799, https://doi.org/ $10.1364 /$ OL.22.000799.

20. Y. Jeong, L. A. Vazquez-Zuniga, S. Lee, and Y. Kwon, On the formation of noise-like pulses in fiber ringcavity configurations, Opt. Fiber Technol. 20 (2014) 575, https://doi. org/10.1016/j.yofte.2014.07.004.

21. S. Kobtsev, S. Kukarin, S. Smirnov, S. Turitsyn, and A. Latkin, Generation of double-scale femto/pico-second optical lumps in mode-locked fiber lasers, Opt. Express 17 (2009) 20707, https://doi.org/10.1364/OE.17.020707.

22. O. Pottiez, R. Grajales-Coutiño, B. Ibarra-Escamilla, E. A. Kuzin, and J. Hernández-García, Adjustable noiselike pulses from a figure-eight fiber laser, Appl. Opt. 50 (2011) E24. https://doi.org/10.1364/AO.50.000E24.

23. R. Ulrich, A. Simon, Polarization optics of twisted single-mode fibers, Appl. Opt. 18 (1979) 2241, https://doi.org/10. $1364 / \mathrm{AO} .18 .002241$.

24. T. Tanemura, K. Kikuchi, Circular-birefringence fiber for nonlinear optical signal processing, J. Lightwave Technol. 24 (2006) 4108, https : //doi.org/10.1109/JLT.2006. 883641 .

25. O. Pottiez, E. A. Kuzin, B. Ibarra-Escamilla, and F. MendezMartinez, Theoretical investigation of the NOLM with highly twisted fibre and a $\lambda / 4$ birefringence bias, Opt. Commun. 254 (2005) 152, https://doi.org/10.1016/j.optcom. 2005.05 .015 .

26. B. Ibarra-Escamilla et al., Experimental investigation of the nonlinear optical loop mirror with twisted fiber and birefringence bias, Opt. Express 13 (2005) 10760, https://doi. org/10.1364/OPEX.13.010760.

27. S. M. J. Kelly, Characteristic sideband instability of periodically amplified average soliton, Electron. Lett. 28 (1992) 806, https://doi.org/10.1049/el:19920508. 